\title{
Personalización política e infoentretenimiento periodístico. Un estudio desde los encuadres
}

\author{
Political personalization and journalistic infotainment. \\ A frame analysis \\ Personalização política e infoentretenimento jornalístico. Um estudo do \\ enquadramento
}

MARTÍN ECHEVERRÍA, Benemérita Universidad Autónoma de Puebla, Puebla, México (echevemartin@yahoo.com.mx]

\section{RESUMEN}

Crecientemente, los medios cubren las elecciones como infoentretenimiento, destacando conflictos y estrategias en lugar de temas y propuestas. La personalización de la política puede explicar en parte esta tendencia, tanto por el diseño de ciertos sistemas políticos, que concentran poder y visibilidad en individuos, como por las narrativas personificantes de los medios. Para indagar su impacto en la cobertura se realizó un análisis comparativo de encuadres vinculados con el infoentretenimiento en la prensa mexicana, entre una elección presidencial altamente personalizada (2012) y una parlamentaria (2015). Los resultados muestran una asociación tenue entre la personalización de una elección y dichos encuadres.

Palabras clave: elecciones; infoentretenimiento; personalización; cobertura; periodismo; encuadres; comunicación política.

\section{ABSTRACT}

News media increasingly cover elections as infotainment, highlighting conflicts and strategies instead of campaign themes and proposals. The personalization of politics could explain this phenomenon, either because of the design of some political systems, that concentrate power and visibility in individuals, or the personalized narratives of the media. To investigate its impact on political coverage, we conducted a comparative content analysis of frames related to infotainment in the Mexican press, between a presidential election, highly personalized, (2012) and a congressional one (2015). Findings show a tenuous association between the personalization of election and such frames.

Keywords: elections; infotainment; personalization; coverage; journalism; frame analysis; political communication.

\section{RESUMO}

Cada vez mais os meios de comunicação cobrem as eleições como infoentretenimento, destacando aspectos de conflito e estratégia em vez de temas e propostas eleitorais. A personalização da política pode ser explicar em parte essa tendência, tanto por desenho de alguns sistemas políticos, que concentram poder e visibilidade em indivíduos, como por as narrativas personificantes dos meios. Para a perguntar sobre seuo impacto na cobertura, uma análise comparativa do infoentretenimiento na imprensa mexicana, entre uma campanha presidencial altamente personalizada (2012), e uma parlamentaria (2015). Os resultados mostram uma associação tênue entre a personalização das eleições e essas variáveis.

Palavras-chave: eleições; infoentretenimento; personalização; cobertura; jornalismo; análise de quadros; comunicação política.

Forma de citar:

Echeverría, M. (2017). Personalización política e infoentretenimiento periodístico. Un estudio desde los encuadres. Cuadernos info, [41], 71-87. https://doi.org/10.7764/cdi.41.1099 


\section{INTRODUCCIÓN}

Desde el punto de vista normativo, la dieta informativa que necesitan los ciudadanos para tomar decisiones electorales debiera llevarlos a conocer y comprender los principales problemas sociales, familiarizarse con los actores políticos postulados para resolverlos y con sus cualificaciones, y estar al tanto de las propuestas y programas que ofertan. No obstante, una parte importante de la ciudadanía carece de las competencias cívicas que le faculten el interés y la comprensión de estos aspectos, razón por la cual ciertos medios se inclinan a elaborar información política que contenga elementos de entretenimiento capaces de atraer a dichas audiencias, a menudo despolitizadas.

De alcance internacional, dicha tendencia periodística ha recibido el nombre de infoentretenimiento, un concepto que prolonga una corriente de reflexiones de larga data centrados en la creciente desacralización, trivialización y popularización de la política y los políticos, en buena medida en virtud de su mediatización (Debray, 1992; Edelman, 2002; Sartori, 1998). A este respecto, la literatura internacional, particularmente la que explora la presencia de encuadres de tipo estratégico y de juego, ha hecho considerables esfuerzos por determinar el alcance y grado de penetración de esa tendencia en diversos países (Berganza, 2008; D’Angelo, Calderone, \&Territola, 2005; Jensen, 2012; Klein, 2000; Semetko \& Valkenburg, 2000; Takens, van Atteveldt, van Hoof, \& Kleinnijenhuis, 2013).

Es menos consensuada, en cambio, una explicación empíricamente validada de dicho fenómeno. Destacan aquellas vinculadas a los constreñimientos y entornos económicos de los medios informativos (Dunaway, 2013; McMenamin, Flynn, O’Malley, \& Rafter, 2012; Rafter, Flynn, McMenamin, \& O'Malley, 2014; Trussler \& Soroka, 2014), a los componentes endógenos de los sistemas mediáticos (Dimitrova \& Kostadinova, 2013; Kerbel, Apee, \&Howard Ross, 2000; Strömbäck \& Dimitrova, 2006; Strömbäck \& Van Aelst, 2010) y, con menor frecuencia, las explicaciones vinculadas a factores específicamente políticos, como la competitividad de las elecciones, la disminución de las diferencias ideológicas o de política pública en los partidos (O’Malley, Brandenburg, Flynn, McMenamin, $\&$ Rafter, 2013), la profesionalización y desarraigo ideológico social de estos (Skorkjær Binderkrantz $\&$ Green-Pedersen, 2009) y la naturaleza consensual o conflictiva del sistema político (Dimitrova \& Kostadinova, 2013)
Notoriamente, uno de los factores políticos menos explorado, aunque más manifiesto, es el de la personalización de la política. Respecto de la visibilidad mediática, es evidente que en una elección federal del Ejecutivo los líderes o personalidades fuertes que pretenden ocupar la figura de presidente o primer ministro alcanzan una prominencia significativa. Aunado a ello, la relevancia cultural de los liderazgos individuales, la naturaleza adversativa o agonal de estos procesos y el concurso de líderes mediáticos carismáticos introduce un componente narrativo que pudiera predisponer un relato más cercano a lo espectacular, estratégico o de juego que, por ejemplo, una elección parlamentaria, plebiscitaria o judicial, donde a nivel formal compiten partidos e instituciones en lugar de personalidades. Existen pues motivos para suponer que el factor de personalización, en su densa significación política y periodística, cultural y narrativa, amolda la cobertura de las elecciones presidenciales a un nivel de espectacularidad que no necesariamente se manifiesta en otro tipo de elecciones o acontecimientos políticos, por lo que su generalización al resto del periodismo político sería equívoca.

Es así que este ejercicio pretende clarificar si la naturaleza de una elección no presidencial y, por lo tanto, no personalizada, a la misma escala nacional, se traduce en variaciones en los patrones de cobertura infoentretenida. A manera de caso de estudio, esto se logra analizando comparativamente la cobertura electoral de la elección presidencial mexicana de 2012 con la legislativa de 2015 y operacionalizando el fenómeno de infoentretenimiento mediante el recurso metodológico de los encuadres, como ya fue mencionado. El marco teórico presentado explicita una definición particular de infoentretenimiento ligada a los encuadres identificados por la literatura y establece el peso previsible de la personalización como un factor diferenciador de la cobertura electoral, vinculado a una narrativa personificadora, a partir de lo cual se generan determinadas hipótesis. La sección metodológica, por su parte, expone los caminos inductivo y deductivo recorridos para establecer el libro de códigos utilizado en el análisis, así como el procedimiento de muestreo. Finalmente, los hallazgos y posteriores conclusiones ponen de manifiesto determinados patrones similares de cobertura centrados sobre todo en las estrategias y los conflictos entre los partidos, así como la exposición mínima de temas, pero con énfasis en estos aspectos vinculados al tipo de elección. 


\section{MARCO TEÓRICO}

PERSONALIZACIÓN POLÍTICA Y NARRATIVAS PERIODÍSTICAS

El fenómeno de la personalización de la política ha constituido una preocupación para los académicos de la comunicación política por lo menos desde el ascenso de la televisión como ventana privilegiada de los actores políticos (Sartori, 1998). No obstante, y a pesar de su ubicuidad, resulta complejo definirlo puesto que en ello se ponen énfasis tanto políticos como mediáticos.

En este primer énfasis la personalización se puede entender como resultado de la organización de jerarquías de poder al interior de los sistemas y organizaciones políticas, que lo distribuyen en mayor medida hacia liderazgos individuales antes que a instituciones o cuerpos colegiados. El diseño básico de los sistemas políticos ilustra esta particularidad: en los sistemas de tipo consensual, una multiplicidad de actores políticos, institucionales y de la sociedad civil tiene un papel en el diseño de políticas públicas; los partidos compiten por el derecho a representar a ciertos segmentos de la sociedad y los cambios en el poder se suscitan a partir de reacomodos en las coaliciones gobernantes, antes que por la vía electoral, lo cual fuerza la cooperación. En contraste, en los sistemas mayoritarios las decisiones tienden a estar centralizadas en un ejecutivo controlado por un solo partido, la representatividad se verifica a escala nacional y las elecciones significan una transferencia casi total del poder a manos de otros actores, de lo que resulta una oposición (de perdedores) a menudo confrontativa (Dimitrova \& Kostadinova, 2013; Hallin \& Mancini, 2004).

En función de esta arquitectura, la atención de los votantes se vuelca en mayor medida en las elecciones presidenciales, consideradas de primer orden, puesto que en estas ponderan sus opciones al atribuirles una mayor importancia en la ejecución de política pública. En contraste, en las elecciones de segundo orden, como las del Congreso, los votantes ponen menos atención y las utilizan sobre todo para expresar insatisfacción con el gobierno o enviarle un mensaje de cambio en la dirección de sus políticas (O’Malley et al., 2013).

Por consiguiente, el diseño político mayoritario y esta particular atracción que ejerce sobre los votantes favorecen lógicamente una mayor personificación del poder, puesto que el sistema les entrega a los líderes políticos márgenes considerables de representatividad y maniobra política. Por consiguiente, la pronunciada visibilidad de los liderazgos personales, durante las elecciones y el gobierno, así como la intensa cobertura mediática con rasgos de confrontación y protagonismo unipersonal en tales periodos, podrían considerarse una respuesta sistémica a tal diseño, en virtud de todo lo que se pone en juego en relación con las personalidades que se eligen y gobiernan (Van Aelst, Sheafer, \& Stanyer, 2012; Vliegenthart, Boomgaarden, \& Jelle, 2011).

Dicho orden institucional interactúa asimismo con una matriz cultural de personalismos que no es, por cierto, privativa de la época contemporánea: la fascinación que despiertan los individuos fuertes y el culto a la imagen personal han sido recursos ampliamente utilizados desde la antigüedad (como en el caso de los Césares romanos), pasando por la primera modernidad (como en el caso de Napoleón) y los sistemas comunistas (Stalin, Castro), en vista de la eficacia emanada del liderazgo personalista. En consecuencia, este rasgo cultural le ha exigido permanentemente a los políticos una dimensión de actuación o puesta en escena que le confiera fuerza y verosimilitud a su discurso (Dader, 1998; Debray, 1992), condición, por cierto, enfatizada recientemente a partir del debilitamiento en la relación afectiva entre votantes y partidos.

Adicional a estos rasgos institucionales y políticoculturales de personalización, la dimensión mediática del fenómeno aporta otros elementos: se observa por un lado la intención estratégica de los actores de incrementar su perfil individual más allá de su membresía partidista, persiguiendo para ello una cobertura periodística intensa y favorable. Por otro lado, los periodistas ejercen criterios que tamizan con tratamientos personificantes la cobertura de temas y acontecimientos políticos, cubren de manera preferente a individuos políticos antes que a instituciones, y enfatizan de estos lo mismo sus cualidades vinculadas a su función pública que sus características personales y de vida privada, irrelevantes para su puesto pero que tienen interés de audiencia (Vliegenthart et al., 2011).

A dicha preferencia anteceden algunos componentes propios de las normas tradicionales de producción periodística: valores de noticiabilidad orientados a individuos antes que grupos, en una jerarquía de poder que apunta invariablemente al máximo nivel del Ejecutivo (Wolf, 2001); la influencia y demandas audiovisuales de la televisión, en virtud de las cuales se prefiere sustituir ideas abstractas y complejas por la gestualidad y discurso concretos de caras reconocibles (Van Aelst et al., 2012), y una propensión a narrar los acontecimientos no desde el punto de vista de los procesos, estructuras e instituciones sociales, sino de individuos inmersos en conflictos y buscando la resolución 
de disputas, con el fin de que estas narrativas produzcan mayor comprensión, identificación e impacto en las audiencias (Johnson-Cartee, 2005).

En suma, los aspectos políticos y mediáticos descritos hacen proclive una cobertura periodística en la que las personas, y no las instituciones o grupos, son los agentes relevantes del acontecer político. Lo característico es que el sistema político personificante se suma a los valores-noticia periodísticos, a partir de lo cual es probable que acentúen determinados patrones contemporáneos de cobertura espectacular, descritos a continuación

\section{ENTRETENIMIENTO POLIITICO PERIODÍSTICO Y ENCUADRES RELATIVOS}

El fenómeno que pretendemos analizar ha adquirido diversas denominaciones en la academia a partir de la dificultad de asirlo teóricamente: a finales de la década de los setenta, las transformaciones en los contenidos periodísticos se han asociado a una lógica mediática (Altheide, 2004); en los noventa, con una tabloidización (Barnett, 1998; Gripsrud, 2000; Zelizer 2009), espectacularización (Lozano, 2004) o noticias suaves (Patterson, 2000); y en los dos mil con el fenómeno del infoentretenimiento, polientretenimiento o política pop (Baym, 2008; Brants, 2005; Mazzoleni \& Sfardini, 2009). Dichas propuestas comparten atributos similares, particularmente la manera en que señalan el descentramiento del periodismo tradicional como discurso privilegiado para comunicar lo político y su consecuente desplazamiento a espacios o lenguajes informales, en buena medida de entretenimiento; no obstante, tienen cualidades diferenciadas que apuntan en varias direcciones.

En consecuencia y como respuesta a esta pluralidad conceptual, el concepto de trabajo para efectos de esta investigación es el de entretenimiento político periodístico ${ }^{1}$, definido como una operación de énfasis, en las noticias políticas, de elementos formales o temáticos capaces de hacer más accesibles y atrayentes los contenidos políticos a audiencias en principio no interesadas o familiarizadas con ese campo. Ello implica que la otrora separación rígida y celosamente guardada entre la información seria y el entretenimiento se diluye y mezcla, poniendo de manifiesto en mayor medida este último (Delli Carpini \& Williams, 2001). También entraña la pretensión de las empresas periodísticas de expandir sus mercados más allá de su habitual audiencia de élite, ya predispuesta al consumo de información política, para lo cual necesita elaborar mensajes capaces de capturar, cautivar y retener la atención de las audiencias, el bien primordial (y escaso) que los medios venden a sus anunciantes (McQuail, 2001).

Ahora bien, la matriz de lenguaje utilizada por la prensa de referencia para hacer estas operaciones corresponde a lo que Altheide (2004) denomina "lógica mediática", procedente originalmente de la televisión y adoptada posteriormente por los tabloides: se trata de una gramática "evocativa, encapsulada, altamente temática, familiar con las audiencias y fácil de usar" (Altheide, 2004, p. 294) que estructura mensajes fragmentarios, simplificados y efectistas para comunicarse con las audiencias de modo eficaz, a la manera de la gramática de la publicidad y el entretenimiento. Esta no solo estructura la presentación del mensaje, sino el resto de los procesos de producción e incluso los supuestos para construir los mensajes; incluye el ritmo, el lenguaje y su formato, "que a su vez prescribe los códigos para definir, seleccionar, organizar, presentar y reconocer información" (Altheide, 2004, p. 294).

La combinación entre los atributos formales mencionados, tales como la brevedad, simplificación, efectismo y lenguaje coloquial, y una selección de temas menos institucionales y más próximos a las audiencias como los escándalos, las noticias útiles o las notas conmovedoras de interés humano, conforma un estilo que se aleja de la complejidad argumentativa y lingüística del periodismo tradicional y se acerca a los formatos televisivos con los que la mayoría de las audiencias están familiarizadas.

Para sintetizar el fenómeno descrito, es posible hacer un recorrido de lo micro a lo macro: en este sentido, la producción de la noticia política es sometida a la lógica mediática, con códigos estilísticos y selecciones temáticas cercanas a la televisión y, por lo tanto, con potencial de interpelación masiva. De esta manera, lo político, otrora serio y argumentativo, se desacraliza, populariza y refina como una mercancía que fundamentalmente pretende llamar la atención de audiencias no educadas o interesadas en la política, bajo un imperativo comercial, lo que termina por relajar la división estable entre información seria y entretenimiento, entremezclándolas y colocando a la primera en primer plano.

En lo que respecta a la operacionalización del concepto, es posible recurrir a la perspectiva teórico metodológica que identifica encuadres o frames ${ }^{2}$ de tipo estratégico o de juego en los mensajes de los medios, a los que es posible añadir otros que responden a un patrón similar, tales como los de conflicto o interés 
humano. Puesto que los encuadres "manifiestan la manera en que las convenciones y procesos de producción periodística traducen los eventos políticos en plantillas para las noticias" (de Vreese, 2014, p. 148), es posible interpretarlos e implementarlos como una expresión simbólica condensada de los recursos utilizados por el infoentretenimiento para atraer la atención de audiencias despolitizadas.

Describimos someramente su contenido: bajo el encuadre de conflicto, la elección se caracteriza como un escenario polarizado, en el cual ocurren fricciones y controversias entre individuos, grupos e instituciones, con poca atención a los temas que están en juego y la substancia de los mismos (Berganza, 2008). Por su parte, el encuadre de juego utiliza el lenguaje y la narrativa de los deportes, y comparte con el anterior los atributos de una competencia férrea, un carácter agonal y confrontativo, así como la expresión de un deseo ferviente por parte de los candidatos de ganar (Anikin, 2009). Es el encuadre que origina el tratamiento de las elecciones como carrera de caballos, con una dosis de especulación acerca de ganadores, perdedores y resultados finales de la carrera (Jensen, 2012; Johnson-Cartee, 2005). En el encuadre estratégico, los reporteros interpretan los motivos e intenciones que subyacen a las acciones o propuestas de los candidatos, así como las tácticas necesarias para que estos ganen posiciones o permanezcan al frente. Describe los eventos de campaña "desde el punto de vista de candidatos interesados en ellos mismos, más que en el bien común” (Patterson, 1993, p. 10). A su vez, el encuadre de interés humano produce relatos que expresan el rostro humano o ángulo emocional de los eventos, temas o problemas relacionados con la campaña, así como acontecimientos graciosos de los candidatos.

En suma, los encuadres reseñados no reportan el contenido político, los temas y problemas públicos ni las propuestas de solución, un desempeño esperado a partir de un entendimiento normativo de los medios como foros de voces plurales que contribuyen a una deliberación política informada (Christians, Glasser, McQuail, \& White, 2009). Antes bien, comunican los resultados parciales de la elección en términos de una contienda dramática, atractiva y excitante; comparten elementos de placer o excitación, el reclamo de atención, lo narrativo en lugar de lo factual y lo popular en oposición a lo formal, sustancioso y, por lo tanto, típicamente elitista. En ese sentido, intentan elevar el nivel de entusiasmo público por los acontecimientos políticos por parte de audiencias despolitizadas, y manifiestan simbólicamente la tendencia de entretenimiento político (Anikin, 2009).

Como mencionamos en la introducción, la producción más o menos intensa de estos patrones de cobertura tiene como condiciones antecedentes, factores de tipo económico, cultural (de cultura periodística) o político. No obstante, dentro de estos últimos, uno que es manifiesto -aunque poco explorado-es el de la personalización de la política, cuya relevancia política, cultural y periodística, con consecuencias inmediatas en términos de cobertura mediática, fue descrita en la primera sección. Tomando dicho factor como variable independiente, es posible esperar un supuesto en relación al entretenimiento periodístico:

- H1. La cobertura de la elección presidencial utilizará en mayor medida los encuadres relacionados con el infoentretenimiento que la cobertura periodística de la elección parlamentaria.

Por otro lado, más allá de las determinaciones del sistema político que enfatizan liderazgos personales, existen actores que realizan esfuerzos deliberados y estratégicos por obtener visibilidad mediática, lo que los puede llevar a colaborar indirectamente con los medios para producir los encuadres vinculados con el infoentretenimiento cuando, por ejemplo, producen declaraciones estratégicas en sus apariciones públicas o sostienen posiciones conflictivas con sus rivales. Si la hipótesis de la personalización es correcta, es probable que aquellos actores individuales estén más expuestos que los partidistas o los gremiales a ser encuadrados de esta manera. En el contexto de una elección, ello genera una segunda hipótesis:

- H2. Los actores políticos individuales, o candidatos, serán cubiertos con encuadres de infoentretenimiento en mayor medida que los actores políticos institucionales.

El diseño de investigación que se expone a continuación apunta pues a esclarecer tales supuestos.

\section{METOdOLOGÍA}

Como caso de estudio, las condiciones que preceden al fenómeno del entretenimiento político periodístico en México, tales como un sistema político que personaliza el poder en virtud de un diseño de tipo mayoritario y presidencialista, elecciones presidenciales -de primer 
orden- más concurridas que las parlamentarias, cierto culto a la personalidad y unos medios informativos modernos inmersos en las formas contemporáneas de hacer periodismo, más narrativas que factuales, justifican en su conjunto la plausibilidad de las hipótesis esbozadas y su investigación empírica. Por otro lado, se dispone de algunos trabajos de naturaleza transversal y descriptiva que dan cuenta de patrones estables de cobertura respecto a cada elección medida, de naturaleza paritaria entre encuadres de política pública y de tipo estratégico o de juego, centrados en la dimensión entretenida de la elección (Echeverría \& Bañuelos, 2015; Echeverría \& Meyer, 2015; Echeverría \& Millet, 2013; Muñiz, 2015).

Si bien el caso mexicano es más bien atípico respecto de otros países latinoamericanos, de modo que sus resultados no serían extrapolables, el diseño y las hipótesis propuestas en dicho escenario pueden servir de punto de partida para replicarse en otras latitudes.

En función de ello, el estudio empírico aquí presentado se concentra en una muestra de notas derivadas de las elecciones federales mexicanas tanto del año 2012 como del 2015, para renovar respectivamente la Presidencia de la República y la Cámara de Diputados. Estos procesos fueron seleccionados por su naturaleza contrafactual respecto de la presencia/ausencia de personalización, componente proclive a tratamientos periodísticos como los ya descritos, así como un contraste sugerente entre elecciones de naturaleza presidencial y parlamentaria. De esta manera, se ponen de manifiesto las ventajas de la comparación en comunicación política, en este caso de contrafactuales, que sensibiliza acerca de las particularidades dadas por sentado por el investigador y hace notar fenómenos que no han sido conceptualizados (Hallin \& Mancini, 2004).

Sin embargo, se debe advertir que se trata de una comparación imperfecta, puesto que está desfasada en el tiempo, lapso durante el cual se pudieron haber introducido variables que posiblemente impacten en los datos comparados. Sin embargo, es necesario observar que una comparación entre elecciones presidenciales y parlamentarias en el mismo año también se dificulta por la manera en que los medios des-enfatizan de su cobertura esta última en favor de la primera, de lo que resulta un contraste muy acentuado. Con todo, esta limitación de validez corresponde a la naturaleza exploratoria de este estudio, aunque deberá ser tomada en cuenta en futuras pesquisas.

El método elegido para llevar a cabo este trabajo es el análisis cuantitativo de contenido. La muestra de periódicos se integró por rotativos correspondientes a la prensa nacional de referencia o de prestigio, debido a su importancia cualitativa en la formación de la opinión pública y por constituir, en teoría, el espacio menos poroso a tendencias de espectacularización, dada la tradición de periodismo liberal que estos rotativos encarnan. Aunque la inclusión de la prensa tabloide o la televisión daría mayor alcance a la hipótesis propuesta, por ser medios de mayor difusión, pensamos que la prensa de referencia demuestra mejor el grado de penetración del infoentretenimiento en el periodismo tradicional, mientras que aquellos medios se han demostrado colonizados por dicha tendencia desde hace varios lustros (Aalberg, Strömbäck, \& de Vreese, 2011).

Por tratarse de rotativos de alto tiraje, distribución nacional, considerados como de prestigio o de referencia, de acuerdo al Padrón Nacional de Medios Impresos (SEGOB, 2013), se revisó el archivo hemerográfico digital de los diarios Reforma, La Jornada, El Universal, Milenio y Excélsior. La muestra se integró por el método de semana compuesta, que consiste en elegir dentro de la población una fecha al azar por cada día de la semana, el cual ha mostrado su confiabilidad estadística en diversos trabajos (Riffe, Lacy, \& Fico, 1998).

La población natural de este ejercicio consistió en todas las ediciones de los cinco rotativos publicadas durante los días que duró la campaña, sesenta en el caso de la parlamentaria, del 5 de abril al 3 de junio de 2015, y noventa en la presidencial, del 30 de marzo al 27 de junio de 2012. Así, en ambos periodos electorales se eligieron siete fechas al azar que contienen 35 ediciones cada una, de lo cual resultaron, para la elección de 2012, 251 unidades de muestreo (notas) y para la del 2015, 172. Solo se eligieron notas informativas que trataran sobre la elección de presidente de la República o de diputados federales, sin considerar piezas de opinión (columnas, editoriales, cartones) o notas de campañas concurrentes a gobernador o presidente municipal.

El libro de códigos utilizado en este ejercicio fue desarrollado para un trabajo anterior y su elaboración combinó procesos inductivos y deductivos. A partir de las categorías y reactivos establecidos por la literatura (Aalberg, Strömbäck, \& de Vreese, 2011; Capella \& Jamieson, 1997; Klein, 2000; Lawrence, 2000; Semetko \& Valkenburg, 2000; Strömbäck \& Luengo, 2008), se realizó un análisis cualitativo por comparación constante a una sub muestra de notas de la elección de 2012 ( $n=212)$ con la finalidad de ajustar aquellas definiciones de los encuadres al contexto mexicano, de lo cual resultaron variaciones mínimas. Si bien ello dificulta 
la comparación de los resultados con las regiones norteamericana y europea, abre el camino a aplicaciones latinoamericanas del método.

Por tanto, dicho libro de códigos contempla dos macro encuadres denominados para este ejercicio como de contienda ${ }^{3}$ y político y siete encuadres generales: interés humano, juego, estrategia y conflicto, correspondiente al primer macro encuadre, y temas, políticas e ideas, proceso político y liderazgo político, al segundo. Para la codificación del corpus específico de este ejercicio, se adoptaron los siguientes criterios: en primera instancia, se decidió identificar un solo encuadre dominante por nota, puesto que a pesar de que la literatura admite la posibilidad de rastrear diversos encuadres en cada pieza, una codificación tan fina dificultaría la obtención de observaciones y conclusiones globales (D’Angelo et al., 2005; Skorkjær Binderkrantz \& Green-Pedersen, 2009). Por su parte, la unidad de análisis dentro de la nota fue el encabezado, por razones prácticas, pero también por razones teóricas, puesto que este constituye el "dispositivo más poderoso de encuadramiento de la nota” (Van Dijk, 1990), que es capaz de activar conceptos en el lector e influenciar así la lectura del resto de la misma (Klein, 2000; Tewksbury, Jones, Peske, Raymond, \& Vig, 2000). Los codificadores clasificaron cada unidad en alguno de los encuadres, utilizando como criterio una relación de tres enunciados descriptivos por cada uno. El hecho de que la unidad ajustara a por lo menos uno de los descriptores era suficiente para clasificarlo en determinado encuadre.

Aplicando la prueba de Kappa de confiabilidad intercodificador sobre el 10\% de las notas de 2012, los macro encuadres tuvieron una confiabilidad de .831 y los encuadres de .739 , considerados satisfactorios para un ejercicio exploratorio como el presente (Riffe et al., 1998). No obstante, en el caso de la elección parlamentaria los datos arrojados en sucesivas pruebas piloto para este ejercicio no lograron pruebas de fiabilidad satisfactorias (.357 y .431, respectivamente, con dos codificadores), posiblemente por un desfase entre el protocolo previamente desarrollado, aplicado a una elección presidencial, y esta de naturaleza legislativa. Sobra decir que dicha tarea es un asunto pendiente en la consecución de un instrumento fiable y replicable en elecciones de distinta naturaleza.

\section{HALLAZGOS}

Un primer resultado a la vez elemental y significativo apunta al volumen de notas recogidas en la muestra y generadas proporcionalmente en cada elección: de las 251 publicadas para cubrir la presidencial se reducen a 172 en la parlamentaria, 1,5 veces menos, lo que expresa con poca ambigüedad un criterio editorial que asigna un valor de noticiabilidad considerablemente menor a estos últimos comicios.

Por otro lado, a nivel de macro encuadres (tabla 1), las proporciones en ambas elecciones varían poco. Para el encuadre de política en 2012 y 2015 hay una diferencia de apenas $0,2 \%$ ( $43,8 \%$ y 43,6\%, respectivamente), que es muy similar para los encuadres de contienda (2012 se ubica en $56,2 \%$ y 2015 , en 56,4\%). Esta falta de variación se apoya con el dato de que no hay significatividad estadística en la relación entre las variables de elección y macroencuadre, $\chi^{2}(1, N=423)=.002, p=.964$, de modo que a este nivel macro, por lo menos, el tipo de elección no parece ejercer una diferencia en su cobertura.

Sin embargo, la frecuencia de los encuadres específicos sí varía significativamente. Si bien el encuadre de liderazgo político se mantiene prácticamente igual, (3,2\% en 2012 y 5,2\% en 2015), dato significativo respecto de la escasa información sustanciosa que en 2012 se publicó sobre los candidatos, la proporción de notas concentradas en el encuadre correspondiente a temas, políticas e ideas tuvo una disminución drástica: en 2012 estos aspectos ocuparon el 19,1\% de las notas, pero para 2015 prácticamente desaparecieron (1,7\%). Tan solo se habló de temas de campaña en tres notas que los tocaron de manera tangencial a algún acontecimiento.

Por lo que respecta al encuadre de proceso político, que trata acerca de los aspectos procedimentales y legales de las elecciones, su proporción en 2015 se duplicó $(36,6 \%)$ respecto de 2012 (21,5\%) particularmente en cuanto a los detalles de organización formal, posiblemente a raíz de la reforma electoral de 2014 que introdujo cambios en los esquemas organizativos y de fiscalización electoral, y que en su momento fueron ampliamente comentados. No obstante, es notorio que la frecuencia de notas es similar en cada elección, 54 en 2012 y 63 en 2015, en lo que parece constituir un tema central de la cobertura mediática.

El análisis de los residuos tipificados corregidos confirma la intensidad de estas asociaciones, puesto que en 2012 la variable temas, políticas e ideas tuvo un valor de 5,4 y en 2015 la de proceso político tuvo un valor de 3,4, mientras que liderazgo político está dentro del rango de +/- 1,96 (1,1), de modo que no hay una asociación entre la elección y esta variable.

Respecto del macro encuadre contienda, los encuadres particulares de cada elección presentan diferencias 
Elección

\begin{tabular}{|c|c|c|c|}
\hline Macro encuadre & & 2012 & 2015 \\
\hline \multirow{3}{*}{ Política } & N & 110 & 75 \\
\hline & $\%$ & 0,44 & 0,44 \\
\hline & Residuos corregidos & 0,0 & 0,0 \\
\hline \multirow{3}{*}{ Contienda } & $\mathrm{N}$ & 141 & 97 \\
\hline & $\%$ & 0,56 & 0,56 \\
\hline & Residuos corregidos & 0,0 & 0,0 \\
\hline \multicolumn{4}{|l|}{ Encuadre } \\
\hline \multirow{3}{*}{ Temas, políticas, ideas } & N & 48 & 3 \\
\hline & $\%$ & 0,19 & 0,02 \\
\hline & Residuos corregidos & 5,4 & $-5,4$ \\
\hline \multirow{3}{*}{ Proceso político } & N & 54 & 63 \\
\hline & $\%$ & 0,22 & 0,37 \\
\hline & Residuos corregidos & $-3,4$ & 3,4 \\
\hline \multirow{3}{*}{ Liderazgo político } & $\mathrm{N}$ & 8 & 9 \\
\hline & $\%$ & 0,0 & 0,1 \\
\hline & Residuos corregidos & $-1,1$ & 1,1 \\
\hline \multirow{3}{*}{ Interés humano } & $\mathrm{N}$ & 14 & 3 \\
\hline & $\%$ & 0,06 & 0,02 \\
\hline & Residuos corregidos & 2,0 & $-2,0$ \\
\hline \multirow{3}{*}{ Juego } & $\mathrm{N}$ & 15 & 5 \\
\hline & $\%$ & 0,1 & 0,0 \\
\hline & Residuos corregidos & 1,5 & $-1,5$ \\
\hline \multirow{3}{*}{ Estrategia } & N & 32 & 8 \\
\hline & $\%$ & 0,13 & 0,05 \\
\hline & Residuos corregidos & 2,8 & $-2,8$ \\
\hline \multirow{3}{*}{ Conflicto } & N & 80 & 81 \\
\hline & $\%$ & 0,32 & 0,47 \\
\hline & Residuos corregidos & $-3,2$ & 3,2 \\
\hline
\end{tabular}

Tabla 1. Comparación entre los encuadres utilizados en cada elección

Fuente: Elaboración propia

contrastantes. En ambos casos, los más prominentes son los de estrategia y conflicto, no obstante, la proporción de ambos varía de manera importante. Mientras que en 2012 el conflicto representaba $31,9 \%$ de la cobertura, en 2015 se elevó a 47,1\%; y si en 2012 la estrategia suponía un $12,7 \%$ de las notas, en 2015 se redujo a un $4,7 \%$, una tercera parte.
Por lo demás, el encuadre de interés humano se redujo en 2012 (5,6\%) y fue prácticamente marginal $(1,7 \%)$ en 2015. Resulta notoria también la baja frecuencia de encuadres de juego en ambas elecciones, relacionado con la difusión de encuestas y el tratamiento deportivo, explícitamente competitivo de la elección, de tan solo 6\% en 2012 y 2,9\% en 2015. 
Tipo de actor

\begin{tabular}{|c|c|c|c|c|c|c|}
\hline Macro encuadre & & Candidatos & Partidos & $\begin{array}{l}\text { Autoridad } \\
\text { electoral }\end{array}$ & $\begin{array}{l}\text { Sociedad } \\
\text { civil }\end{array}$ & $\begin{array}{l}\text { Otras } \\
\text { fuentes }\end{array}$ \\
\hline \multirow{3}{*}{ Política } & $N$ & 84 & 25 & 38 & 8 & 30 \\
\hline & $\%$ & 0,45 & 0,33 & 0,66 & 0,25 & 0,42 \\
\hline & Residuos corregidos & 0,4 & $-2,0$ & 3,6 & $-2,2$ & $-0,3$ \\
\hline \multirow{3}{*}{ Contienda } & $N$ & 103 & 50 & 20 & 24 & 41 \\
\hline & $\%$ & 0,55 & 0,67 & 0,34 & 0,75 & 0,58 \\
\hline & Residuos corregidos & $-0,4$ & 2,0 & $-3,6$ & 2,2 & 0,3 \\
\hline \multicolumn{7}{|l|}{ Encuadre } \\
\hline \multirow{3}{*}{ Temas, políticas, ideas } & $N$ & 49 & 0 & 0 & 0 & 2 \\
\hline & $\%$ & 0,26 & 0,00 & 0,00 & 0,00 & 0,03 \\
\hline & Residuos corregidos & 8,0 & $-3,5$ & $-3,0$ & $-2,2$ & $-2,6$ \\
\hline \multirow{3}{*}{ Proceso político } & $N$ & 29 & 20 & 37 & 6 & 25 \\
\hline & $\%$ & 0,16 & 0,27 & 0,64 & 0,19 & 0,35 \\
\hline & Residuos corregidos & $-5,0$ & $-0,2$ & 6,6 & $-1,2$ & 1,6 \\
\hline \multirow{3}{*}{ Liderazgo político } & $\mathrm{N}$ & 6 & 5 & 1 & 2 & 3 \\
\hline & $\%$ & 0,03 & 0,07 & 0,02 & 0,06 & 0,04 \\
\hline & Residuos corregidos & $-0,8$ & 1,3 & $-1,0$ & 0,7 & 0,1 \\
\hline \multirow{3}{*}{ Interés humano } & $N$ & 10 & 1 & 1 & 1 & 4 \\
\hline & $\%$ & 0,05 & 0,01 & 0,02 & 0,03 & 0,06 \\
\hline & Residuos corregidos & 1,2 & $-1,3$ & $-1,0$ & $-0,3$ & 0,8 \\
\hline \multirow{3}{*}{ Juego } & $\mathrm{N}$ & 11 & 2 & 0 & 2 & 5 \\
\hline & $\%$ & 0,06 & 0,03 & 0,00 & 0,06 & 0,07 \\
\hline & Residuos corregidos & 1,0 & $-0,9$ & $-1,8$ & 0,4 & 1,0 \\
\hline \multirow{3}{*}{ Estrategia } & $N$ & 22 & 5 & 2 & 2 & 9 \\
\hline & $\%$ & 0,12 & 0,07 & 0,03 & 0,06 & 0,13 \\
\hline & Residuos corregidos & 1,4 & $-0,9$ & $-1,7$ & $-0,6$ & 1,0 \\
\hline \multirow{3}{*}{ Conflicto } & N & 60 & 42 & 17 & 19 & 23 \\
\hline & $\%$ & 0,32 & 0,56 & 0,29 & 0,59 & 0,32 \\
\hline & Residuos corregidos & $-2,3$ & 3,5 & $-1,5$ & 2,6 & $-1,1$ \\
\hline
\end{tabular}

Tabla 2. Relación entre actores y encuadres

Fuente: Elaboración propia.

En general, estas diferencias marcadas en los encuadres, y de manera señalada en los de conflicto, estrategia, proceso político y temas, le confieren significatividad estadística a la relación entre tipo de elección y encuadres, $\chi^{2}(6, N=423)=54.114, p=<.001$, incluso con una vinculación moderada ( $V$ de Cramer, $.358, p=<.001)$ entre ambas variables. Los residuos tipificados corregidos prácticamente descartan asociaciones importantes entre las elecciones y las variables de interés humano (2,0 en 2012) y juego (1,5 en 2012), y confirman una relación moderada entre la elección presidencial de 2012 y el encuadre de estrategia $(2,8)$, así como la parlamentaria de 2015 y la variable de conflicto $(3,2)$. 


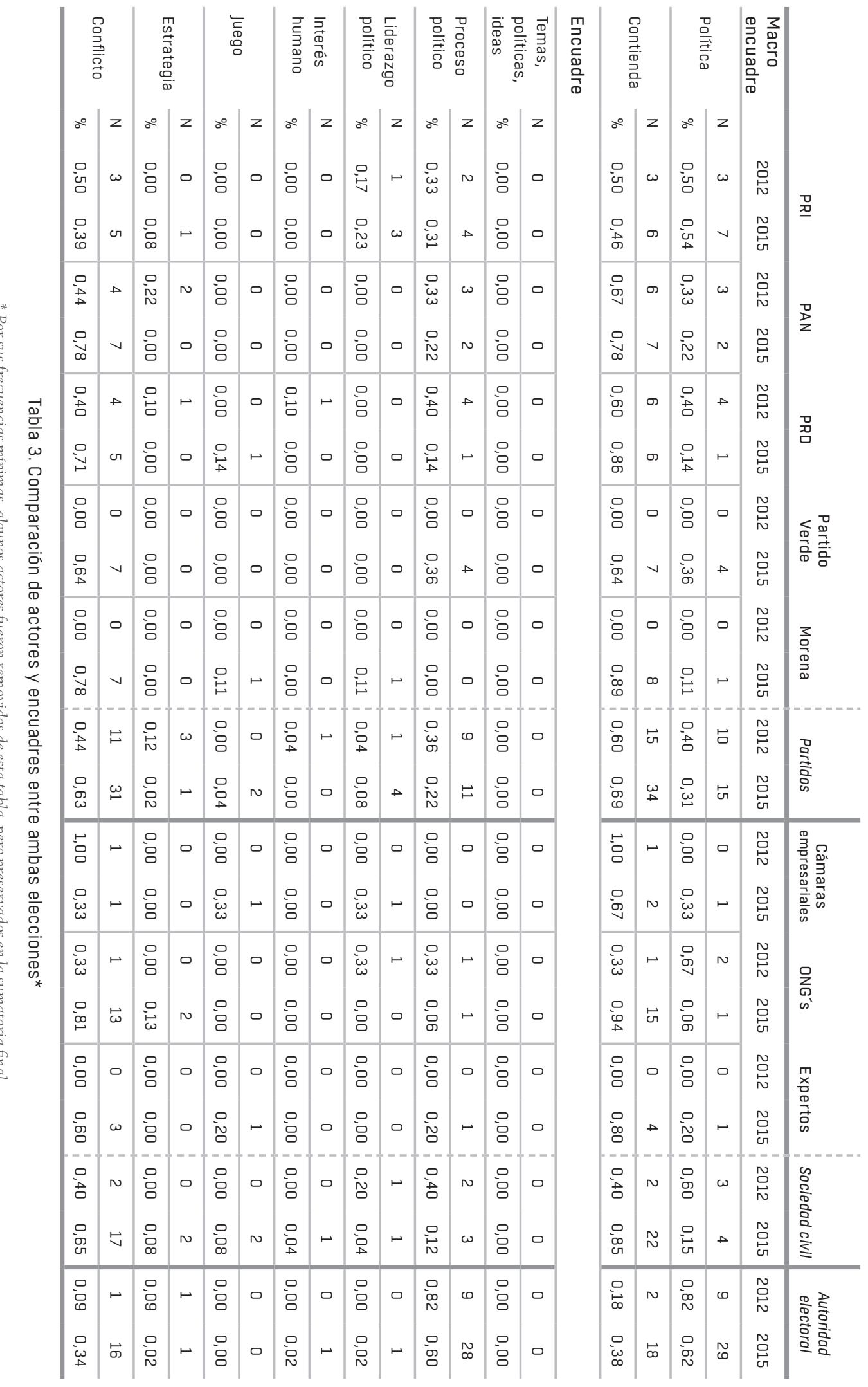


La exploración de la segunda hipótesis se despliega en la tabla 2, en donde los 25 actores que fungieron como fuentes en las distintas notas fueron condensados en cuatro tipos de ellas, de acuerdo a sus similitudes. Reportamos de cada una de ellas el porcentaje de cobertura, así como el valor del residual corregido tipificado, en ese orden. Contrario a lo esperado, los candidatos no se asocian con el macro encuadre contienda $(55 \%,-4)$, mientras que los partidos y la sociedad civil sí lo hacen (67\% y $75 \%$, respectivamente, 2 y 2,2 en residuos corregidos). Esta particularidad es más clara a nivel de encuadres particulares, en donde los candidatos no están asociados ni al encuadre de juego $(6 \%, 1)$ y estrategia $(12 \%, 1,4)$, y apenas están vinculados con el encuadre de conflicto $(32 \%,-2,3)$. Por el contrario, los candidatos están fuertemente asociados a temas, políticas e ideas $(26 \%, 8)$ y en buena medida al "proceso político" $(16 \%,-5)$.

De manera sugerente, son los partidos políticos y la sociedad civil los que tienen mayor asociación con el macro encuadre de contienda ( $67 \%$ y $75 \%$, respectivamente, y 2 y 2,2 en residuos corregidos), así como una mayor vinculación con el encuadre de conflicto (56\% y $59 \%, 3,5$ y 2,6 en residuos corregidos). En general, los datos presentan una relación estadísticamente significativa entre el tipo de actor cubierto y macro encuadre, $\chi^{2}(4, N=423)=19.215, p=001$, pero con una correlación pequeña $(\mathrm{V}$ de $C r a m e r=.213, p=<.001)$, así como una relación estadísticamente significativa entre la primera variable y el encuadre específico, $\chi^{2}(24, N=423)=$ $125.556, p=<.001$, aunque con una correlación esta vez moderada (V de Cramer $=.272, p=<.001)$. Esto implica que la intensidad de uso de los encuadres está relacionada con el tipo de actor de que se trate, sin ser una tendencia general, aunque no es aplicable a los candidatos.

Un último conjunto de datos compara la cobertura de los actores entre elección y elección, y aunque tiene una cualidad más descriptiva, pudieran dale mayor contexto a la segunda hipótesis. Si bien algunos de estos actores no son comparables debido a la naturaleza de las contiendas, como por ejemplo el tratamiento de los candidatos, dada la ínfima proporción de notas acerca de dichos actores en el proceso 2015 en comparación con la presidencial de 2012, ello no sucede de dicha manera con otros tres agrupamientos de actores bien diferenciados: partidos políticos, autoridad electoral (Instituto Nacional Electoral, Tribunal Electoral del Poder Judicial de la Federación) y sociedad civil (expertos, organizaciones empresariales, confesionales y no gubernamentales) (tabla 3).
Así, en términos de macro encuadres, los partidos políticos reciben un tratamiento inesperadamente similar en ambas elecciones, tomando en cuenta que su visibilidad incrementa notablemente en las legislativas (de 25 a 49 notas), ocupando probablemente el espacio de los candidatos presidenciales en el 2012. No obstante, el tratamiento contencioso en ambos casos es predominante en ambas elecciones, en una proporción de dos terceras partes de las notas (60\% en 2012 y $69,4 \%$ en 2015), incrementando ligeramente en la última elección.

Respecto de los encuadres específicos, dos de ellos predominan mientras que los otros tienen frecuencias mínimas: las noticias de los partidos son encuadradas con mayor frecuencia como un proceso político en 2012 (36\%) que en $2015(22,4 \%)$ y sobre todo como un asunto de conflicto, agudizado en 2015 (63,3\%) respecto de 2012 (44\%).

Por otro lado, la autoridad electoral, representada en este caso por el INE y el Tribunal Electoral del Poder Judicial, incrementó notablemente su visibilidad en la cobertura periodística e ingresó a una caracterización adversativa vis a vis con los partidos políticos y la sociedad civil: de tan solo 11 notas publicadas en 2012, pasó en 2015 a 47 de ellas, un incremento considerable. El macro encuadramiento general de contienda se duplicó de 18,2\% a 38,3\% para dicho actor, y el encuadre de conflicto pasó de 9,1\% a 34\% de las notas. Esto, naturalmente, redujo el encuadre de proceso político de $81,8 \%$ en 2012 a 59,6\% en 2015.

Los organismos de la sociedad civil recibieron una cobertura mucho mayor en 2015 (26 notas) que en 2012 (5). Si bien tanto las cámaras empresariales o la Iglesia Católica incrementaron su visibilidad, fueron las ONG quienes más cobertura recibieron en comparación con el proceso de 2012, pues de tres notas pasaron a 16. El tratamiento de estos grupos sociales incrementó notablemente su énfasis en aspectos de entretenimiento, puesto que pasó de 40\% de las notas en 2012 a 84,6\% en 2015. En particular, la información relacionada con los organismos de la sociedad civil incrementó su encuadramiento en términos de conflicto, de 33,3\% en 2012 a 81,3\% en 2015.

Llama la atención, finalmente, que los periódicos de la muestra no encuadraron la información electoral proveniente de estos tres conjuntos de actores como un asunto de temas, políticas e ideas, aspecto que sí se verifica en cambio para los candidatos de 2012. Este vacío no es menor, dado que, al menos normativamente, se supone que los medios fungen como vehículos de las 
problemáticas y propuestas de campaña, útiles a las necesidades de información de las audiencias en su carácter de ciudadanos.

\section{CONCLUSIONES}

Esta investigación se propuso confirmar hipótesis respecto de la influencia de la personalización política, tanto en su énfasis propiamente político como mediático, en la cobertura periodística vinculada con el infoentretenimiento. Los resultados indican que la personalización de una elección presidencialista, de carácter agonal tanto en su naturaleza política como en su mediatización, no parece estar relacionada con una cobertura de encuadres relacionados con el infoentretenimiento. También indican que la personificación de un actor determinado, en comparación con actores grupales y más abstractos, tampoco es un factor que incremente la probabilidad de recibir dicho tratamiento. En suma, la personalización no explica la prevalencia de encuadres de infoentretenimiento.

Por otra parte, las conclusiones de este estudio van en sentido contrario a otros de naturaleza similar en elecciones europeas, donde la personalización se confirma como una explicación diferenciadora de la cobertura, aunque ahí los sistemas mayormente parlamentarios pudieran ser el factor clave de tales discrepancias. (Dimitrova \& Kostadinova, 2013; Skorkjær Binderkrantz \& Green-Pedersen, 2009). Ello refuerza la necesidad de realizar estos estudios con mayor énfasis en sistemas presidencialistas, como los de varios países latinoamericanos.

Este estudio tiene ciertas limitaciones que pudieran dar luz sobre el sentido de los resultados. Respecto de su capacidad explicativa, la variable independiente está considerablemente simplificada puesto que solo contempla una dimensión de personalización, la naturaleza del tipo de elección de que se trata, excluyendo otros indicadores del mismo fenómeno, además de que está circunscrita a un solo caso de estudio, el mexicano. Asimismo, no involucra diversas elecciones de una u otra naturaleza, de modo que los resultados bien pudieran estar asociados a diversos factores coyunturales relacionados con el comportamiento reciente de los medios, antes que a un fenómeno estructural; esta misma limitación aplica a la asincronía en la comparación ente ambas elecciones (2012 y 2015), en cuyos años de diferencia pudieron presentarse modificaciones en las condiciones políticas o mediáticas relativas a la personalización. De igual manera, los problemas observados con la fiabilidad de los datos de la elección de 2015 dificultan elaborar aseveraciones concluyentes, de modo que las relaciones evidenciadas deberán interpretarse con cautela. Todas estas advertencias pudieran ser tomadas en cuenta en futuras investigaciones de este tipo.

\section{DISCUSIÓN}

Un primer indicio del influjo de la personalización en la cobertura pudiera ser el dato de la mayor cantidad de notas publicadas en la elección presidencial en comparación con la parlamentaria. Aunque es difícil discernir si es un efecto del valor-noticia de jerarquía o bien de la personalización política, una lectura sistémica admite interpretar que el particular arreglo del sistema político mexicano, de naturaleza presidencialista y por tanto mayoritaria, termina por conferirle mayor relevancia periodística a la elección del poder Ejecutivo.

Por lo que respecta a la primera hipótesis comparativa, acerca de que la cobertura periodística de la elección presidencial utilizará en mayor medida los encuadres relacionados con el infoentretenimiento que la cobertura periodística de la elección parlamentaria, esta no se apoya por los datos. En primer lugar y a un nivel de macro encuadres, se verifica una relativa paridad entre los encuadres de política y de contienda, tanto al interior de cada elección y entre ambas elecciones. Los datos constatan una presencia significativa, aunque no predominante, de esta forma de cobertura infoentretenida en la prensa mexicana, en coincidencia con otros trabajos (Echeverría \& Bañuelos, 2015; Echeverría \& Meyer, 2015; Echeverría \& Millet, 2013; Muñiz, 2015). Puesto que dicha paridad se sostiene entre ambas elecciones, este comportamiento pudiera poner de manifiesto un rasgo estructural de la prensa mexicana.

Asimismo, al observar los datos acerca de los encuadres específicos en cierta manera la hipótesis propuesta se invierte por partida doble: por un lado, el encuadre de conflicto se incrementa de manera importante en las parlamentarias, al pasar de una tercera parte $(31,9 \%)$ a cerca de la mitad $(47,1 \%)$ de las notas y, por otro lado, el encuadre contrario al infoentretenimiento, el de temas, políticas e ideas, se reduce drásticamente de 19,1\% en 2012 a 1,7\% en 2015.

En ese sentido, son las elecciones parlamentarias las que producen un tratamiento más cercano al infoentretenimiento que las presidenciales. Esta afirmación se contextualiza al tomar en cuenta el tratamiento de los actores involucrados en la cobertura. Si bien a 
nivel de macro encuadres se incrementa ligeramente una cobertura contenciosa, el encuadre de conflicto se eleva notoriamente de 2012 a 2015, lo mismo para partidos políticos (de $44 \%$ a $63 \%$ ), autoridad electoral (de $9,1 \%$ a $34 \%$ ) y organizaciones de la sociedad civil (de 33\% a $81 \%$, respectivamente), al tiempo que dichos actores no son cubiertos bajo el encuadre sustancioso referente a temas de campaña y propuestas políticas.

Por otro lado, la segunda hipótesis establecía que los actores individuales, en virtud de su personalización, serían mayormente encuadrados en un sentido de infoentretenimiento que los grupales. Los datos tampoco apoyan esta hipótesis: apuntan, de hecho, a una asociación mayor de los candidatos con los temas y propuestas, y también corroboran que los partidos políticos y la sociedad civil, entidades grupales, son más proclives a tratamientos de infoentretenimiento. Ambos resultados demuestran incluso que los factores de personalización, como las elecciones presidenciales y los candidatos, se asocian con coberturas periodísticas más bien tradicionales y políticamente sustanciosas.

A pesar de las limitaciones metodológicas expuestas previamente, los hallazgos a nivel exploratorio son sugerentes y aquellos, junto con el diseño teórico metodológico utilizado, creemos que sientan las bases para la realización de estudios longitudinales y comparativos en un mismo sistema, así como extrapolados a otros distintos, que permitan establecer con mayor precisión los factores relacionados con las formas de cobertura periodística entretenida.

NOTAS

1. La denominación de "entretenimiento político" aún enfrenta el problema de definir un proceso de generación de contenidos por su efecto, como si la acción de estos fuera forzosamente entretener a las audiencias. No obstante, resuelve la necesidad de colocarlo en una línea de investigación de objetos afines, a pesar de lo arbitrario de su nomenclatura.

2. Para efectos de este trabajo, los encuadres se comprenden como esquemas de tratamiento de la información periodística que se manifiestan en la selección, exclusión y énfasis de ciertos observables sobre otros, y su organización en un discurso que los presenta en función de un determinado significado (Muñiz, 2011; Reese, 2001; Zhang, 2000). Los encuadres residen tanto internamente en los periodistas, como guías internalizadas de procesamiento de información, como externamente en los contenidos periodísticos (Klein, 2000) y están gobernados por constricciones institucionales, prácticas o ideologías (Sádaba, Rodríguez, \& Bartolome, 2007).

3. La literatura suele denominar al macro encuadre contrario al político como estratégico. No obstante, y derivado de nuestros análisis, nos parece que este tipo de cobertura excede elementos de estrategia política al contemplar aspectos de emotividad o conflicto, por lo cual dicho término se queda corto en relación a lo que designa. La expresión de contienda, en cambio, es una metáfora centrada en la dimensión agonal de la competencia electoral, que engloba con mayor facilidad los aspectos ya mencionados. 


\section{REFERENCIAS}

Aalberg, T., Strömbäck, J., \& de Vreese, C. H. (2011). The framing of politics as strategy and game: A review of concepts, operationalizations and key findings. Journalism, 13(2), 162 -178. https://doi.org/10.1177/1464884911427799

Altheide, D. (2004). Media Logic and Political Communication. Political Communication, 21(3), 293-296. https://doi.org/10.1080/10584600490481307

Anikin, E. E. (2009). The 2008 US Presidential Election in the Mirror of Sports Metaphor (in the french press). Respectus Philologicus, 16(21), 46-55. Retrieved from https://www.ceeol.com/search/articledetail?id=224148

Barnett, S. (1998). Dumbing Down or Reaching Out: Is it Tabloidisation wot done it? The Political Quarterly, 69(B), 75-90. https://doi.org/10.1111/1467-923x.00193

Baym, G. (2008). Infotainment. In W. Donsbach (Ed.), The International Encyclopedia of Communication. Malden: Blackwell Publishing (Blackwell Reference Online. 15 November 2017, http://www.communicationencyclopedia.com).

Berganza, M. R. (2008). Medios de comunicación, "espiral del cinismo" y desconfianza política. Estudio de caso de la cobertura mediática de los comicios electorales europeos [Mass Media, "the Spiral of Cynicism" and Political Distrust. Case Study of Media Coverage of European Elections]. Zer: Revista de Estudios de Comunicación, 13(25), 121-139. Retrieved from http://www.ehu.eus/zer/es/hemeroteca/ articulo/Medios-de-comunicacin-espiral-del-cinismo-ydesconfianza-poltica-Estudio-de-caso-de-lacoberturameditica-de-los-comicios-electorales-europeos/369

Brants, K. (2005). Quem tem medo do infotainment? [Who's afaid of infotainment]. Media \& Jornalismo, 7(7), 39-58. Retrieved from http://fabricadesites.fcsh.unl.pt/polocicdigital/wp-content/uploads/ sites/8/2017/03/n7-02-Kees-Brants.pdf

Capella, J. \& Jamieson, K. H. (1997). Spiral of Cynicism: The Press and the Public Good. Oxford: Oxford University Press.

Christians, C. G., Glasser, T., McQuail, D., \& White, R. (2009). Normative Theories of the Media. Journalism in Democratic Societies. Illinoils: University of Illinoils Press.

D’Angelo, P., Calderone, P., \& Territola, A. (2005). Strategy and Issue Framing: An Exploratory Analysis of Topics and Frames in Campaign 2004 Print News. Atlantic Journal of Communication, 13(4), 199 219. https://doi.org/10.1207/s15456889ajc1304_1

Dader, J. (1998). Tratado de comunicación política [Treatise of political communication]. Madrid: Edicion propia.

Debray, R. (1992). L'État séducteur. Les révolutions mediologiques du pouvoir. Paris: Editions Gallimard.

Delli Carpini, M. X. \& Williams, B. A. (2001). Let Us Infotain You: Politics in the New Media Age. In L. Bennet \& R. Entman (Eds.), Mediated Politics. Communication in the Future of Democracy (pp. 160-181). Cambridge: Cambridge University Press.

de Vreese, C. H. (2014). Mediatization of News: The Role of Journalistic Framing. In F. Esser \& J. Strömbäck (Eds.), Mediatization of Politics: Understanding the Transformation of Western Democracies (pp. 137-155). London: Palgrave Macmillan UK.

Dimitrova, D. \& Kostadinova, P. (2013). Identifying Antecedents of the Strategic Game Frame: A Longitudinal Analysis. Journalism \& Mass Communication Quarterly, 90(1), 75-88. https://doi.org/10.1177/1077699012468739

Dunaway, J. (2013). Media Ownership and Story Tone in Campaign News. American Politics Research, 41(1), 24-53. https://doi.org/10.1177/1532673x12454564

Echeverría, M. \& Bañuelos, R. (2015). El infoentretenimiento en los procesos parlamentarios. El caso de la reforma energética [Infotainment in parlamentary processes. The case of the energy reform]. In B. Chong (Ed.), Perspectivas de la Comunicación en 2015. A 22 años de Investigación en el CONEICC [Perspectives of Communication in 2015. 22 years of Research in CONEICC] (pp. 41-60). Mexico: Consejo Nacional para la Enseñanza y la Investigación de las Ciencias de la Comunicación. 
Echeverría, M. \& Meyer, J. A. (2015). El infoentretenimiento en la cobertura periodística de las elecciones. Un abordaje desde la teoría del encuadre [Infotainment in the coverage of elections. A framing approach]. In D. Ivoskus, A. Mendieta, \& R. Marina (Eds.), Sexto sentido para gobernar. Política y comunicación [Sixth sense to govern. Politics and communication] (pp. 211-230). Puebla: BUAP, Paralelo Cero.

Echeverría, M. \& Millet, A. (2013). El “infoentretenimiento” en campaña. El caso de los debates presidenciales de 2012 [Infotainment in campaign. The case of the 2012 presidential debates]. In C. Vidal (Ed.), XX Anuario de la Comunicación CONEICC [XX Yearbook of Communication CONEICC] (pp. 235-253). Mexico: CONEICC.

Edelman, M. (2002). La construcción del espectáculo político [The construction of political spectacle]. Buenos Aires: Manantial.

Gripsrud, J. (2000). Tabloidization, Popular Journalism and Democracy. In C. Sparks \& J. Tulloch (Eds.), Tabloid Tales. Global debates over media standards (pp. 285-300). Lanham: Rowman and Littlefield Publishers.

Hallin, D. C. \& Mancini, P. (2004). Comparing Media Systems. Three Models of Media and Politics. Cambridge: Cambridge University Press.

Jensen, L. C. (2012). Politics as a Game in Danish Newspapers. Sonderborg Papers in Linguistics and Communication, (2), 1-10. Retrieved from http://www.sdu.dk/ /media/Files/Om_SDU/Institutter/Ifki/ SPLC/Student\%20files/Politics\%20as\%20a\%20Game\%20in\%20Danish\%20Newspapers.pdf

Johnson-Cartee, K. (2005). News narratives and news framing. Constructing political reality. Oxford: Rowman and Littlefield.

Kerbel, M. R., Apee, S., \& Howard Ross, M. (2000). PBS Ain't So Different: Public Broadcasting, Election Frames, and Democratic Empowerment. The Harvard International Journal of Press/Politics, 5(4), 8-32. https://doi.org/10.1177/1081180X00005004002

Klein, U. (2000). Tabloidized Political Coverage in the German Bild-Zeitung. In C. Sparks \& J. Tulloch (Eds.), Tabloid tales. Global debates over media standards (pp. 177-195). Lanham: Rowman and Littlefield Publishers.

Lawrence, R. (2000). Game-Framing the Issues: Tracking the Strategy Frame in Public Policy News. Political Communication, 17(2), 93-114. https://doi.org/10.1080/105846000198422

Lozano, J. C. (2004). Espectacularización de la información en noticieros televisivos de Canadá, Estados Unidos y México [Spectacularization of information in the newscasts of Canada, the United States and Mexico]. Diálogo Político, 21(1), 101-116. Retrieved from https://psisocom0910.files.wordpress. com/2009/10/espectacularizacion_de_la_informacion.pdf

Mazzoleni, G. \& Sfardini, A. (2009). Politica pop: da "Porta a porta" a "Lisola dei famosi" [Pop politics: from "Door to Door" to "The Island of the famous]. Bologna: Il mulino.

McMenamin, I., Flynn, R., O’Malley, E., \& Rafter, K. (2012). Commercialism and Election Framing: A Content Analysis of Twelve Newspapers in the 2011 Irish General Election. The International Journal of Press/Politics, 18(2), 167-187. https://doi.org/10.1177/1940161212468031

McQuail, D. (2001). Introducción a la teoría de la comunicación de masas [Introduction to the theory of mass communication]. Madrid: Paidos.

Muñiz, C. (2011). Encuadres noticiosos sobre migración en la prensa digital mexicana. Un análisis de contenido exploratorio desde la teoría del framing [News approaches on migration in Mexican digital press; an analysis of exploratory nature from the framing theory]. Convergencia, 18(55), 213-239. Retrieved from https://convergencia.uaemex.mx/article/view/1138

Muñiz, C. (2015). La política como debate temático o estratégico. Framing de la campaña electoral mexicana de 2012 en la prensa digital [Politics as an issue or strategic debate. Framing of the 2012 Mexican electoral campaign in the digital press]. Comunicación y Sociedad, (23), 67-95. Retrieved from http://www.comunicacionysociedad.cucsh.udg.mx/index.php/comsoc/article/view/64/83 
O’Malley, E., Brandenburg, H., Flynn, R., McMenamin, I., \& Rafter, K. (2013). The impact of the economic crisis on media framing: evidence from three elections in Ireland. European Political Science Review, 6(3), 407-426. https://doi.org/10.1017/s1755773913000155

Patterson, T. (1993). Out of Order. New York: Vintage.

Patterson, T. (2000). Doing Well and Doing Good. How Soft News and Critical Journalism Are Shrinking the News Audience and Weakening Democracy-And What News Outlets Can Do About It. Boston: John F. Kennedy School of Government, Harvard University.

Rafter, K., Flynn, R., McMenamin, I., \& O’Malley, E. (2014). Does commercial orientation matter for policy-game framing? A content analysis of television and radio news programmes on public and private stations. European Journal of Communication, 29(4), 433-448. https://doi. org/10.1177/0267323114532204

Reese, S. (2001). Prologue. Framing public life. In S. Reese, O. Gandy, \& A. Grant (Eds.), Framing public life. Perspectives on media and our undestanding of the social world. Oxon: Routledge.

Riffe, D., Lacy, S., \& Fico, F. (1998). Analyzing Media Messages. Mahwah, NJ: Taylor \& Francis Group.

Sádaba, T., Rodríguez, J., \& Bartolome, M. (2007). Propuesta de sistematización de la teoría del framing para el estudio y praxis de la comunicación política [Proposal for the systematization of framing theory for the study and practice of political communication]. Observatorio (OBS), 6(2), 109-126. https://doi.org/10.15847/obsOBS622012540

Sartori, G. (1998). Homo videns. La sociedad telediriga [Homo videns. The teledirected society]. Madrid: Taurus.

Secretaría de Gobernación (SEGOB). (2013). Padrón Nacional de Medios Impresos [National Catalog of Print Media]. SEGOB. Retrieved from http://pnmi.segob.gob.mx/.

Semetko, H. A. \& Valkenburg, P. M. (2000). Framing European Politics: A Content Analysis of Press and Television News. Journal of Communication, 50(2), 93-109. https://doi.org/10.1111/j.1460-2466.2000.tb02843.x

Skorkjær Binderkrantz, A. \& Green-Pedersen, C. (2009). Policy or Processes in Focus? The International Journal of Press/Politics, 14(2), 166-185. https://doi.org/10.1177/1940161209333088

Strömbäck, J. \& Dimitrova, D. (2006). Political and Media Systems Matter A Comparison of Election News Coverage in Sweden and the United States. The International Journal of Press/Politics, 11(4), 131147. https://doi.org/10.1177/1081180X06293549

Strömbäck, J. \& Luengo, Ó. (2008). Polarized Pluralist and Democratic Corporatist Models A Comparison of Election News Coverage in Spain and Sweden. International Communication Gazette, 70(6), 547-562. https://doi.org/10.1177/1748048508096398

Strömbäck, J. \& Van Aelst, P. (2010). Exploring Some Antecedents of the Media's Framing of Election News: A Comparison of Swedish and Belgian Election News. International Journal of Press/Politics, 15(1), 41-59. https://doi.org/10.1177/1940161209351004

Takens, J., van Atteveldt, W., van Hoof, A., \& Kleinnijenhuis, J. (2013). Media logic in election campaign coverage. European Journal of Communication, 28(3), 277-293. https://doi.org/10.1177/0267323113478522

Tewksbury, D., Jones, J., Peske, M. W., Raymond, A., \& Vig, W. (2000). The Interaction of News and Advocate Frames: Manipulating Audience Perceptions of a Local Public Policy Issue. Journalism \& Mass Communication Quarterly, 77(4), 804-829. https://doi.org/10.1177/107769900007700406

Trussler, M. \& Soroka, S. (2014). Consumer Demand for Cynical and Negative News Frames. The International Journal of Press/Politics, 19(3), 360-379. https://doi.org/10.1177/1940161214524832

Van Aelst, P., Sheafer, T., \& Stanyer, J. (2012). The personalization of mediated political communication: A review of concepts, operationalizations and key findings. Journalism, 13(2), 203-220. https://doi.org/10.1177/1464884911427802 
Van Dijk, T. (1990). La noticia como discurso: comprensión, estructura y producción de la información [News as discourse: understanding, structure and production of information]. Barcelona: Paidos.

Vliegenthart, R., Boomgaarden, H. G., \& Jelle, W. B. (2011). Changes in Political News Coverage: Personalization, Conflict and Negativity in British and Dutch Newspapers. In K. Brants \& K. Voltmer (Eds.), Political Communication in Postmodern Democracy Challenging the Primacy of Politics. London: Palgrave Macmillan.

Wolf, M. (2001). La investigación de la comunicación de masas [Mass media research]. Mexico: Paidos.

Zelizer, B. (2009). Tabloidization: What is it and Does it Really Matter? In B. Zelizer (Ed.), The changing faces of journalism. Tabloidization, technology and truthiness. New York: Routledge.

Zhang, W. (2000, August). An interdisciplinay synthesis of framing. Paper presented at the Association for Education in Journalism and Mass Communication, Anual Meeting, Phoeniz, Arizona. Retrieved from http://list.msu.edu/cgi-bin/wa?A3=ind0101b\&L=AEJMC\&E=quoted-printable $\& \mathrm{P}=8547234 \& B$

SOBRE EL AUTOR

Martín Echeverría Victoria, profesor-investigador del Instituto de Ciencias de Gobierno y Desarrollo Estratégico, Benemérita Universidad Autónoma de Puebla, México. Doctor en Comunicación y Cultura [Universidad de Sevilla], Magister en Comunicación Política y Opinión Pública (Universitat Pompeu Fabra, Barcelona). Miembro del Sistema Nacional de Investigadores, nivel 1. Sus líneas de investigación son los formatos audiovisuales de la comunicación política, los estudios de periodismo político y la relación entre consumo mediático, jóvenes y política. 